\title{
Does orthopaedic resident efficiency improve with respect to decreased fluoroscopic times in tibial intramedullary nailing? A measure of an ACGME milestone
}

Bradburn, Kayla MD; Patel J. H. MD; Cannada, Lisa K. MD

\section{INTRODUCTION}

Tibial fractures are common traumatic orthopaedic injuries that are often treated with fluoroscopy guided intramedullary nailing. The Accreditation Council for Graduate Medical Education (ACGME) has included intramedullary nailing of tibia fractures and effective use of imaging as surgical milestones for orthopaedic surgery residents. However, there are no documented quantifiable methods to measure these competencies. ${ }^{1}$ Previous studies have shown a correlation between increased surgeon experience and decreased fluoroscopy usage, but fluoroscopy times in intramedullary nailing have not been assessed. ${ }^{2-6}$

In this retrospective review, we sought to characterize duration of fluoroscopic usage during tibial intramedullary nailing by resident post graduate year (PGY) and by time of year (first half of academic year versus second half). We hypothesized we would note improved fluoroscopic times during tibial intramedullary nailing by residents during their advancement through their orthopaedic residency and in the second half of the academic year compared to the first half.

\section{MATERIALS AND METHODS}

This is the author's manuscript of the article published in final edited form as:

Bradburn, K., Patel, J., \& Cannada, L. (2019). Does orthopaedic resident efficiency improve with respect to decreased fluoroscopic times in tibial intramedullary nailing? A measure of an ACGME milestone. Current Orthopaedic Practice, 30(2), 129-132. https://doi.org/10.1097/BCO.0000000000000733 
This was a retrospective cohort study at two level one Trauma Centers. The two centers are served by residents and attending trauma staff from a single institution. After Institutional Review Board approval, Current Procedural Terminology (CPT) codes were used to identify patients treated with intramedullary nailing of Orthopaedic Trauma Association (OTA) 42A \& 42B tibia fractures under the direction of fellowship trained trauma staff between January 2007 December 2014. Patients with both closed and open fractures were included. We evaluated resident levels PGY2-5 who completed tibial shaft intramedullary nailing surgeries during the specified time. Exclusion criteria were patients undergoing concomitant fluoroscopic procedures, poor radiographic/procedural records, any attending supervising the procedure who had not completed an orthopaedic trauma fellowship, or surgeries involving primary assisting residents with less than two post graduate years.

The data collected included patient BMI, age, timing of academic year of surgery (first half defined as July-December, second half as January-June), fracture classification, number of screws, total fluoroscopic imaging time in seconds, and PGY level of the operating resident. All attending staff were fellowship trained orthopaedic trauma surgeons. Their years in practice were also included in data analysis.

Descriptives and chi-square were used for nominal data and t-tests for continuous variables. We compared overall groups between half years and then looked at junior residents (PGY-2 and PGY-3) compared to senior residents (PGY-4 and PGY-5) for each of the continuous variables. A Bonferroni correction was utilized to evaluate multiple t-tests. IBM SPSS version 23.0 was used. 


\section{RESULTS}

\section{Demographics}

345 total patients were identified. After exclusions, 159 fractures were included in 154 patients. Five patients had bilateral tibia fractures that were treated with intramedullary nailing. The majority of exclusions were due to lack of fluoroscopic times and procedures with only attendings and PGY-1 residents. 41 female patients and 113 males were included. Average patient age was 37.6 years.

\section{Fracture details}

The fractures were classified according to the OTA Fracture classification and there were 3942 $A 1,6042 A 2,2342 A 3,642 B 1,2142 B 2$, and $1943 B 3$ fractures.

\section{Surgical Results}

47 tibias were operated on by junior residents: 43 were operated on by PGY-2s, four were operated on by PGY-3s. 112 tibias were operated on by senior residents: 27 were operated on by PGY-4s, and 85 were operated on by PGY-5s. The distribution of fracture types was not significantly different among resident groups, $p=0.381$. Fracture type and body mass index had no effect on fluoroscopic use, $\mathrm{p}=0.459, \mathrm{p}=0.968$ respectively. The relationship between open or closed fractures and residents' operative fluoroscopic times was insignificant, $p=0.741$. This relationship was also insignificant when considering attendings' years of experience, $\mathrm{p}=0.858$. Poller blocking screws were used in 24 of the operations. The number of interlocking screws or 
use of Poller blocking screws had no significance with fluoroscopic usage in any year of training or when comparing a particular year to others, $\mathrm{p}>0.05$.

\section{First half of academic year}

The first half of the academic year had no recorded tibial nailing performed primarily by a PGY3 resident. Therefore, their evaluation is not included. Mean operative times for PGY-2, PGY-4, and PGY-5 residents were 1.84, 1.71, and 1.98 hours respectively. When compared individually or grouped as juniors and seniors, all operative time differences were insignificant.

Operative fluoroscopic usage times, ranges, and 95\% confidence intervals are summarized in Table 1 for each post graduate year. When comparing PGY-2 residents to all other residents using an independent samples t-test, they completed tibial nailing slowest in terms of fluoroscopic usage than any other years, $p=0.003$. Also, when comparing PGY-4 residents to all other residents, they completed tibial nailing significantly faster in terms of fluoroscopic usage than any other years, $\mathrm{p}=0.031$. PGY -4 residents used less fluoroscopy than PGY-2 and PGY $-5 \mathrm{~s}$, $\mathrm{p}=0.041, \mathrm{p}=0.041$.

\section{Second half of academic year}

Mean operative times for PGY-2, PGY-3, PGY-4, and PGY-5 residents were 1.88, 1.69, 1.62, and 1.93 hours respectively. When compared individually or grouped as junior and seniors, all operative time differences were insignificant. 
Operative fluoroscopic usage times, ranges, and $95 \%$ confidence intervals are summarized in Table 2 for each post graduate year. PGY-5 residents used significantly less fluoroscopy than PGY-2 residents, $\mathrm{p}=0.035$. All other comparisons between PGYs were insignificant. 
When grouped as juniors and seniors and compared by whole academic year, the senior group used significantly less fluoroscopy, $\mathrm{p}=0.018$. 47 Juniors had a mean usage of 258.30 seconds, and 112 seniors had a mean usage of 207.39 seconds. There was no difference in attending years of experience and fluoroscopic use.

\section{DISCUSSION}

Although the ACGME has published surgical milestones for orthopaedic surgery residents, they offer no objective measures to aid in evaluation of resident physicians. For example, under the "Diaphyseal Femur and Tibia Fracture- Patient Care" section, one of the criteria is "Effectively uses intraoperative imaging." "While this is currently evaluated through subjective views of resident performance, our research suggests that this may instead be objectively evaluated through looking at fluoroscopic times.

Diaphyseal tibia fractures are common orthopedic injuries and often treated with intramedullary nails. Fluoroscopic imaging provides surgeons with a better understanding of fracture reduction and aids in hardware placement. It has been suggested that inexperienced residents use more fluoroscopic imaging during these operative procedures, which our data supports. ${ }^{2-5}$

Additionally, fluoroscopic imaging carries an inherent radiation risk, and efforts should be made to minimize exposure to radiation. Surgeons and OR personnel are especially vulnerable due to their cumulative radiation exposure, and risk cataracts, thyroid dysfunction, cancer, and 
reproductive health issues secondary to this exposure..$^{7-10}$ Therefore, fluoroscopic usage is an ideal metric to monitor resident proficiency because in addition to correlating with resident years of experience, it is also beneficial to minimize.

Patient factors do not appear to play a role in fluoroscopic usage. Notably, we examined the relationship between BMI and fracture types on fluoroscopy time and both were found to have an insignificant relationship. This is interesting and useful for evaluating residents' use of fluoroscopy, as our data demonstrates patient and fracture characteristics should not influence fluoroscopic usage. To our knowledge, this is the first study with these findings and shows fluoroscopic usage can be applied independent of patient factors to the evaluation of residents' performance and progression through their years of training. However, prospective studies are needed to ensure there is no confounding variable such as increased attending involvement in specific fracture patterns or in cases with obese patients.

Although we specifically studied tibial nailing, other milestones also may be objectively evaluated using fluoroscopic usage. Other ACGME surgical milestones that commonly use fluoroscopy during fixation are distal radius fractures, adult elbow fractures, pediatric supracondylar humerus fractures, and hip fractures. ${ }^{1}$ These milestones may also be able to use fluoroscopic proficiency in residents' evaluation, but further studies are needed. While the ACGME has established the milestones project to evaluate residents' progression during residency using the aforementioned injuries, their evaluation is only subjective in nature. Objective measurements would likely be beneficial to the ACGME milestones project and the 
evaluation of residents' performance. The addition of fluoroscopy use would provide another data point, one that is uniquely objective.

As work hour restrictions have in some cases limited the amount of operative cases available to residents, other methods of training may be of particular importance to help ensure adequate training. ${ }^{11}$ Thorough preoperative planning and simulation should be considered in the surgical training of the orthopaedic residents as these modalities may ultimately assist in reduced radiation exposure to the patient, surgeons, and staff. While there is strong agreement among residents and residency program directors that orthopaedic skills simulators should be a mandatory component of residency training, substantial barriers still exist. ${ }^{12}$ One of the main obstacles is identifying reliable and valid metrics to determine the impact and efficacy of the simulation based training. ${ }^{13}$ Our research suggests that fluoroscopic usage could be one such metric used to gauge improvement and skill progression. This metric could be applied to not only tibial intramedullary nail simulators but also several other procedures that involve intramedullary nailing and fluoroscopy.

This study does have limitations. We had few numbers of PGY-3s recorded performing tibial shaft nailing. This in part can be due to the set rotation schedule of the institution. PGY-3s only perform trauma at one institution along with a PGY-4. Therefore if both were involved in any particular case, only the PGY-4 would be recorded as performing the surgery as they were the most senior resident. The lack of data on PGY 3 residents also introduced the possibility of bias that PGY-4s and PGY-5s were being compared with PGY-2s. Another limitation was the possibility that other residents may have assisted in the surgery. While the most senior resident in 
the case was recorded as the operative resident, the senior may have permitted the junior to take the role of first assist during surgery under his or her supervision. Therefore, some senior residents' fluoroscopic usage may have been inflated due to the junior's use of fluoroscopy. We believe that this may account for the unexpected finding that PGY-4's used the least amount of fluoroscopy instead of PGY-5's, since it was common for PGY-5's to be coupled with PGY-2's. In a retrospective study, there was no reliable way to account for this possibility, as the amount of involvement of a junior under a senior is a very subjective decision, and even surgical logs cannot adequately determine. However, a prospective approach may be able to minimize this limitation.

Our study had numerous strengths. One was the number of patient cases included. 159 tibias were evaluated, and we believe this amount is enough to draw appropriate conclusions. The majority of cases involved attending surgeons who had more than five years' experience outside of an orthopaedic trauma fellowship. This level of experience leads to the consistency in operative procedures and training of residents.

\section{CONCLUSION}

When grouped appropriately, senior residents performed tibial nailing faster than junior residents, including the last half of the academic year. As the ACGME currently has no measurements for resident progress and efficiency regarding tibial shaft nailing, our data is encouraging that fluoroscopic measurements may be useful in assessing resident proficiency. Fluoroscopic monitoring may aid programs in accurately assessing resident progress and help set 
quantifiable goals that may be applied to the current ACGME orthopaedic milestones project. Future studies should look to externally validate fluoroscopic usage as a metric for resident proficiency, and possibly use a prospective approach to address some of the limitations of our study.

\section{CONFLICTS OF INTEREST AND SOURCES OF FUNDING}

There are no funding sources to disclose or conflicts of interest. 


\section{REFERENCES}

1. Orthopaedic Surgery Milestones, ACGME Report Worksheet. 15 Acta Orthop. 2015 Apr 17:1-6. [Epub ahead of print]

2. Botchu R, Ravikumar K. Radiation exposure from fluoroscopy during fixation of hip fracture and fracture of ankle: Effect of surgical experience. Indian J Orthop. 2008;42(4):471-3.

3. Kheiran A, Makki D, Banerjee P, Ricketts D. Intraoperative radiation use by trainees during ankle fracture fixation. Orthopedics. 2013;36(9):1165-8.

4. Tsalafoutas IA, Tsapaki V, Kaliakmanis A, et al. Estimation of radiation doses to patients and surgeons from various fluoroscopically guided orthopaedic surgeries. Radiat Prot Dosimetry. 2008;128(1):112-9.

5. Blattert TR, Fill UA, Kunz E, et al. Skill dependence of radiation exposure for the orthopaedic surgeon during interlocking nailing of long-bone shaft fractures: a clinical study. Arch Orthop Trauma Surg. 2004;124(10):659-64.

6. Kirousis G, Delis H, Megas P, Lambiris E, Panayiotakis G. Dosimetry during intramedullary nailing of the tibia. Acta Orthop. 2009;80(5):568-72.

7. Madan S, Blakeway C. Radiation exposure to surgeon and patient in intramedullary nailing of the lower limb. Injury. 2002;33(8):723-7.

8. Tasbas BA, Yagmurlu MF, Bayrakci K, Ucaner A, Heybeli M. Which one is at risk in intraoperative fluoroscopy? Assistant surgeon or orthopaedic surgeon?. Arch Orthop Trauma Surg. 2003;123(5):242-4. 
9. Chou LB, Chandran S, Harris AH, Tung J, Butler LM. Increased breast cancer prevalence among female orthopedic surgeons. J Womens Health (Larchmt). 2012;21(6):683-9.

10. Chow R, Beaupre LA, Rudnisky CJ, Otto D, Clark M. Surgeons' perception of fluoroscopic radiation hazards to vision. Am J Orthop. 2013;42(11):505-10

11. Nasca TJ, Day SH, Amis ES. The new recommendations on duty hours from the ACGME Task Force. N Engl J Med. 2010;363(2):e3.

12. Karam MD, Pedowitz RA, Natividad H, Murray J, Marsh JL. Current and future use of surgical skills training laboratories in orthopaedic resident education: a national survey. J Bone Joint Surg Am. 2013;95(1):e4.

13. Atesok K, MacDonald P, Leiter J, et al. Orthopaedic education in the era of surgical simulation: Still at the crawling stage. World J Orthop. 2017;8(4):290-294. 